\title{
EDITORIAL
}

\section{Darwinian molecular imaging}

\author{
Bertrand Tavitian • Uwe Haberkorn
}

Published online: 26 June 2009

(C) Springer-Verlag 2009

On 12 February 2009, we celebrated the 200th anniversary of Charles Darwin's birthday. His theory of evolution through natural selection [1] is a most famous biological concept, with a notoriety as wide as Albert Einstein's concept of relativity in physics. If, as Dobzhansky provocatively stated, "Nothing in biology makes sense except in the light of evolution" [2], then it is worthwhile that we, as molecular imaging practitioners and scientists, should ask ourselves how much Darwinian is molecular imaging today. What use of Darwin's theory of evolution through natural selection is made in the fields of molecular imaging and nuclear medicine? Is the evolution theory conceptually useful or a mere theoretical notion occasionally discussed by basic researchers in molecular imaging? Could the introduction of Darwinian concepts be operant to evolve more efficient and better molecular imaging? Now 200 years after his birth and 150 years after the first edition of his famous book, one of the greatest successes in scientific publishing ever (the first edition sold out in just 1 day), it appears timely for molecular imaging to take stock of Darwin's heritage.

B. Tavitian

Inserm U803, and Service Hospitalier Frédéric Joliot,

Institut d'Imagerie Biomédicale,

CEA,

Orsay, France

U. Haberkorn $(\bowtie)$

Department of Nuclear Medicine, University Hospital Heidelberg, and Clinical Cooperation Unit Nuclear Medicine,

DKFZ,

Heidelberg, Germany

e-mail: Uwe.Haberkorn@med.uni-heidelberg.de

\section{Darwin's theory of evolution through natural selection}

In 1859, Charles Darwin published On the Origin of Species by Means of Natural Selection, or the Preservation of Favoured Races in the Struggle for Life [3]. The theory of natural selection results from insightful connections between a huge corpus of meticulous observations of living species, both animals and plants, wild and domesticated, collected during 30 years of scientific activity.

Several exegetes (see among others Ernst Mayr, Dobzhansky, Patrick Tort) have unveiled the logics of construction of Darwin's theory, which we briefly summarize here in four principles:

1. Principle of variability: both wild and domestic animals exhibit phenotypic variation; hence, living species have a natural propensity towards variation.

2. Principle of the limitation of population size: the reproduction capacity of most species is so high that, in the absence of limitation, they would grow exponentially. This is not observed and population sizes generally tend to be stable because a regulatory factor limits population sizes.

3. Principle of selection: breeders and farmers select variations that they deem advantageous by allowing only those individuals with desired traits to produce an offspring. Several of these traits are transmitted to the offspring.

4. Principle of the survival of the fittest: similar to the limitation of population sizes by breeders, competition for survival is observed throughout natural populations. Only individuals that have inherited the genetic traits best adapted to their environment survive and produce offspring. Therefore, in a given environment the driving force for selection is the struggle for life and only the best fit to that environment survive. 
Over very long scales of time (evolutionary ages), favourable inherited traits gradually accumulate in populations, and this natural selection can lead to the appearance of a new species. This is the origin of species.

A theory of the evolution of species, a phenomenon that generally requires more than the lifetime of an experimenter, is not easily accessible to the experimental method. However, Darwin's theory provides a rich framework that biologists constantly confront with their results. It is remarkable that his theory should have stood up so well (except possibly for the gradual aspect of the changes that underlie the origin of species) to the many tests it has been subjected to, even after the subsequent discovery of genetics and molecular biology. Indeed, the notion of the gene fits in perfectly with Darwin's idea of variation, as does Mendelian segregation with the genetic inheritance of traits. The discovery of the role of nucleic acids in the transmission of hereditary characters, and the determination of the double-helix structure of DNA, almost immediately provided a virtually perfect biochemical explanation for the transmission of inherited characters.

\section{Darwinian approaches to ligand molecular imaging probe selection}

Whatever fascination one may have for the beauty of Darwin's theory, it can be argued that the first, essential principle of Darwinism, i.e. the variability of individuals among an offspring, is irrelevant to the radiotracer principle [4]. Obviously, a tracer (radioligand or other) administered in vivo with the aim of imaging a biological target does not reproduce itself while travelling through the body and therefore bears no offspring that can experience variability, selection or adaptability. Hence, at first sight, molecular imaging appears to have little, if any, connection with Darwin's theory, apart from the apparently minor point that the environment exerts a selective pressure on the "survival" of the tracer, i.e. its escape from body excretory and metabolic processes.

Combinatorial approaches that select the best candidates among a panel of molecular imaging probes are not unusual for radioligand selection prior to administration, for instance by derivatization of common labelled motifs in order to improve targeting, stability and bioavailability: among classic examples are the optimization of RGD-based tracers for integrin imaging, of somatostatin analogues for the SSR2 receptor and of radioligands targeting the PBR-TSOP receptor for neuroinflammation. However, these approaches are not Darwinian in the sense that probe variants are generated and selected independently of heredity and reproduction.

In that respect, the only biological molecules that can generate "offsprings" are the polymeric aperiodic crystals
[5] based on macromolecular polymers of amino acids or nucleotides. Through the manipulation of cells and enzymes that produce these macromolecules, biotechnology has developed fabrication processes that closely resemble natural mechanisms of reproduction, but without requiring the presence of a living being. This mastering of the living tool box has already produced many entities with industrial prospects, including artificial nano-objects with properties unknown in nature [6-8]. New probes can now be fabricated by an artificial adaptation of natural molecular synthesis mechanisms for the production of macromolecules with excellent recognition capacity. Some of these Darwinian combinatorial approaches to generation and selection of binding macromolecules are tempting for use in molecular imaging. In those cases the scientist is responsible for designing the variations in the pool of the species, i.e. molecules, that have to encompass and survive the selection pressure imposed on them. And he is also creating the varying environment with changing selection pressures to reach the goal of selecting specifically interacting molecules. Although this procedure has a touch of creationism, the mechanisms at work are purely based on Darwinian processes.

\section{Molecular evolution of oligonucleotide binders (aptamers)}

Long after Darwin hypothesized a physical support for heredity that he called "gemmules", it was demonstrated that nucleic acids are the chemical support for heredity because of their capacity to replicate through Watson-Crick pairing of nucleobases [9]. In addition, nucleic acids can fold into a myriad of structures selected by natural evolution for catalytic activity [10] or for specific interaction with proteins $[11,12]$ or small molecules. These two properties of nucleic acids, the ability of a mother sequence to generate descendants and the capacity of a sequence to interact with other molecules, led to the invention of a technology designed to evolve populations of nucleic acids iteratively, by the selection, at each successive generation, of those structures best suited for a given property [13, 14]. This selective evolution of ligands by exponential enrichment (SELEX) [14] produces aptamers, a neologism coined from the Latin aptus, meaning "apt" or "appropriate for". The basic tenets of the Darwinian theory of evolution consubstantial to aptamers and SELEX, i.e. the generation of populations of individuals and the selection of the most apt individuals, are reflected in another appellation of SELEX, directed molecular evolution.

The SELEX methodology has proved remarkably fertile for the production of aptamers with medical or technological applications including molecular imaging. Some 
imaging applications appear unique to the nucleic acid structure of aptamers as, for instance, their capacity to fold into a stable spatial structure in the presence of their ligand ("structuration on the ligand" [15]) which is now routinely employed to turn aptamers into beacons producing a fluorescent signal upon structural rearrangement in the presence of the target [16]. In this latter embodiment, a fluorophore is attached to an aptamer and a quencher to the aptamer's complementary sequence; binding of the target releases quenching and generates a fluorescent signal [16]. RNA aptamer sequences that bind fluorophores and are expressed inside cells are used to report on the regulation of an expression system. Studies by the 2008 Nobel prize winner Roger Tsien [17, 18] and others [19] have shown that the insertion of an aptamer sequence in the non-coding region of a mRNA can provide a means to control and document the expression of that particular mRNA upon addition of the relevant target of the aptamer.

Aptamers have also been engineered to report on targeted drug delivery by a combination of three elements: (1) a fluorescent quantum dot (QD) as scaffold, (2) the anticancer fluorescent drug doxorubicin and (3) an aptamer targeting the prostate-specific membrane antigen (PSMA) protein as cancer targeting agent. In the double-quenched "off" state, fluorescence from the QD is quenched by doxorubicin, while fluorescence from doxorubicin is quenched by the aptamer. Upon delivery into prostate cancer cells, doxorubicin is released from the aptamer, unquenching the fluorescent signal from both the drug and the QD and reporting on intracellular delivery of the drug by the PSMA aptamer [20].

\section{In vivo nuclear imaging with aptamers}

Aptamers are attractive as molecular imaging probes because of their medium molecular size (10-20 kDa), limited steric hindrance for binding to targets in complex environments and high selectivity of target recognition. Indeed, their capacity to discriminate selectively their target inside a mixture has been reported to be superior to that of antibodies: an aptamer targeting neutrophil elastase showed a target to background ratio superior to that of the reference antibody for imaging inflammation in vivo [21]. More recently, an aptamer raised against human tenascin-C was labelled with ${ }^{99 \mathrm{~m}} \mathrm{Tc}$ and, after injection into nude mice bearing tumour xenografts of human U251 glioma, yielded a tumour to blood ratio of 50 at $3 \mathrm{~h}$ and 180 at $16 \mathrm{~h}$, suggesting the possible use of aptamers as imaging agents to recognize cancer targets in vivo [22, 23]. However, even if aptamers conceptually represent a direct validation of the theory presented by Darwin 150 years ago, more studies are necessary to establish aptamers as imaging agents. Questions to be addressed include the influence of the labelling group on aptamer biodistribution, the tradeoff between uptake level and washout for a better image contrast, the binding mechanism and the minimal concentration of a target that can be recognized by aptamers in vivo. Fortunately, aptamers are generated by a synthetic selection process in which the experimental setting may be fully controlled for the fabrication of ligands endowed with specific recognition characteristics. Hence, there is large room for their improvement, for instance through multimerization or conjugation to nano-scaffolds, and many possibilities are available to introduce changes in aptamer structure through defined chemical modifications or for conjugation to a wide array of labels or other molecules. Aptamers can address a very wide range of targets, even those that are too toxic to be used as antigens for raising antibodies in a living organism, as well as targets that do not elicit antibody response.

As far as pharmacological applications are concerned, the time required for the development of aptamers from their invention in the laboratory to their first registration as FDA approved drugs (Macugen ${ }^{\mathrm{TM}}$ [24]), i.e. 13 years, is in the same order as required for the development of a "standard" medicine, which suggests that we may be right at the beginning of the entry of these "Darwinian nanoobjects" into molecular medicine.

\section{Display systems for the identification of high-affinity binders}

Display systems are used for the selection of molecules from libraries in which peptides or proteins are physically linked to their corresponding encoding sequences. In addition, these systems can be applied to modify the biophysical properties of the displayed molecules by evolution through cycles of mutation, selection and replication. In principle, two types of display systems are available: cell-based systems such as phage display or cell surface display and cell-free systems such as ribosome display and mRNA display.

\section{Phage display}

The principle of phage-displayed protein or peptide libraries is the display of these libraries fused with the carboxy-terminal domain of the minor coat protein (gene III or VII protein fragment) on the surface of a filamentous phage $[25,26]$. The relevant molecule is then directly detected and screened using the target molecules and amplified after infection with Escherichia coli. This allows the rapid selection of particular clones from large pools 
$\left(>10^{10}\right.$ clones) and determination of the amino acid sequence of a protein/peptide displayed on a phage by sequencing the relevant section of the phage genome. Selection is done by exposing the library to the target proteins or cells or even injecting it into animals (biopanning). The method is used for various applications, such as mapping and mimicking of epitopes, identifying new receptors and natural ligands, identifying high-affinity antibodies and analogues, isolating specific antigens that bind to bioactive compounds, producing novel enzyme inhibitors and DNA-binding proteins and probing cellular and tissue-specific processes. Phage display was successfully applied for the identification of novel peptides with high specificity $[27,28]$ and has been employed to isolate an NG2 proteoglycan-binding peptide to target tumour neovasculature [29] or to identify specifically binding peptides for human lung carcinoma, mammary carcinoma, prostate carcinoma and neuroblastoma cells [30-34].

Human antibody fragment libraries displayed on phages are obtained by cloning of $\mathrm{V}_{\mathrm{H}}$ and $\mathrm{V}_{\mathrm{L}}$ domains of human antibodies in filamentous phage DNA and displaying them on the surface coat of phages as single-chain Fv or Fab fragments. This procedure results in the isolation of highaffinity human antibodies (at the nanomolar range) of many different antigens within a few weeks. For the production of recombinant single-chain $\mathrm{Fv}$ fragments $(\mathrm{scFv})$, the $\mathrm{V}_{\mathrm{H}}$ and $\mathrm{V}_{\mathrm{L}}$ domains are linked together with a polypeptide bridge (about 15 hydrophilic amino acid residues) and screened by phage display. However, these monovalent antibody fragments are cleared rapidly from the blood as a result of their small molecular size and their monodenticity. Since intact antibodies are generally polyvalent molecules, many attempts have been made to combine Fab or scFv molecules into dimers or higher multimers such as diabodies, triabodies and minibodies to produce highly functional reagents of $60-120 \mathrm{kDa}$ in size. Diabodies and triabodies show rapid tumour penetration, are relatively flexible from the orientation of antibody-binding sites and reveal a higher functional affinity with reduced kidney clearance rates. Minibodies have proven to be efficient in localizing tumour xenografts in mice and show high retention in tumour cells [35].

\section{Imaging and therapy with molecules identified by phage display}

Using antibody phage display libraries a single-chain Fv antibody (L19) fragment was isolated, which recognized an epitope of the ED-B domain of fibronectin, a marker of angiogenesis, with a dissociation constant of $0.054 \mathrm{nM}$. Animal experiments with L19 showed targeting of this molecule to F9 murine teratocarcinoma. More than 20\% ID/ $\mathrm{g}$ of the dimeric L19 accumulated in the tumour at $4 \mathrm{~h}$ postinjection (p.i.) with tumour to organ ratios up to 8.6 at $4 \mathrm{~h}$ and 29.4 at $24 \mathrm{~h}$ after tracer administration [36]. In a study with 20 patients with lung, colorectal or brain cancer scintigraphy with the ${ }^{123}$ I-labelled dimeric L19 localized in tumour lesions in aggressive types of lung cancer and colorectal cancer [37]. The molecule was also used in preclinical studies for tumour detection after labelling with ${ }^{76} \mathrm{Br}$ [38] or ${ }^{99 \mathrm{~m}} \mathrm{Tc}$ [39]. Finally, radioimmunotherapy in two colorectal tumour models resulted in tumour growth inhibition and improved survival of the tumour-bearing animals [40].

Furthermore, a peptide binding to galectin-3 (gal-3), a carbohydrate-binding protein that has been implicated in cell adhesion, tumour invasion and metastasis were identified by phage display [41] and evaluated in a mouse model of metastatic human prostate carcinoma expressing gal-3. The ${ }^{111}$ In-labelled peptide bound with a $50 \%$ inhibitory concentration of $191 \pm 10.2 \mathrm{nM}$ to $\mathrm{PC} 3-\mathrm{M}$ prostate carcinoma cells. In vivo tumour uptake was moderate with $1.27 \%$ $\mathrm{ID} / \mathrm{g}$ at $30 \mathrm{~min}$ and associated with a fast whole-body clearance.

Using a 12-mer peptide library a variety of peptides for prostate cancer (DUP-1), thyroid cancer (FROP-1), head and neck cancer (HBP-1) and hepatoma (E7) were identified [31, 34, 42]. Specific binding was verified by competition experiments. Internalization occurred in some of these peptides (DUP-1, FROP-1 and HBP-1) indicating a possible therapeutic use. Interestingly, FROP-1 binds also to a variety of other cell lines which can be seen as evidence that this peptide can be applied as a pan-tumour agent. Stability was a problem with short half-lives between 2 and $55 \mathrm{~min}$ for these molecules. Therefore, one of the peptides (FROP-1) was coupled to the chelator DOTA which resulted in structural restriction and consequently improved binding and stability. When compared to the free peptide without chelator, FROPDOTA revealed different cellular uptake kinetics reaching a maximum at $2 \mathrm{~h}$ in vitro. The cells completely accumulated the tracer and competition experiments showed almost $100 \%$ inhibition of tracer accumulation (in FRO82-2, MCF-7 and others). The internalization kinetics determined in MCF-7 cells supported this finding: after an incubation time of $180 \mathrm{~min}$ the major fraction of FROPDOTA was trapped intracellularly. Serum stability experiments revealed an increase in stability due to the chelator, with a half-life of $71 \mathrm{~min}$. Circular dichroism measurements indicated a fixed alpha helix structure of FROPDOTA representing a strong change in secondary structure. In competition binding experiments the binding constant $\left(\mathrm{K}_{\mathrm{D}}\right)$ to FRO82-2 cells was determined to be $494 \mathrm{nM}$. Despite this avid binding affinity the binding kinetics was found to be too slow to induce an uptake in vivo prior to clearance. Consequently, the biodistribution 
revealed a rapid renal and hepatobiliary clearance with blood levels dropping from $5.48 \pm 0.26 \% \mathrm{ID} / \mathrm{g}$ at $5 \mathrm{~min}$ p.i. to $0.77 \pm 0.15 \% \mathrm{ID} / \mathrm{g}$ at $135 \mathrm{~min}$ p.i.

\section{Bacterial display}

In the bacterial FliTrx ${ }^{\mathrm{TM}}[43,44]$ system a phagemid vector (pFliTrx) allows the display of peptides directly on the surface of $E$. coli by utilizing two proteins: the major bacterial flagellar protein (FliC) and thioredoxin (TrxA). Peptide libraries are cloned in frame within the active site loop of thioredoxin which is inserted into the dispensable region of the FliC gene. The resulting fusion protein assembles into the flagella on the bacterial cell surface protruding from the cells. The dodecamer peptide library is constrained by a disulphide bridge, which should result in a stable conformation of the synthetic peptide as well as an increased resistance to degradation.

The bacterial peptide display system was used to identify a new prostate carcinoma-binding peptide (MM-2) that is stable in serum and binds to prostate carcinoma cells in vitro but shows a low affinity for benign prostate cells. Binding to HUVECs was weaker and not significantly inhibited by unlabelled MM-2, indicating specificity of the peptide toward prostate tissue [45]. MM-2 has no sequence similarity to bombesin, luteinizing hormone-releasing hormone, prostate-specific antigen or any other peptide or protein sequence available, as confirmed by a search of various protein databases such as the European Molecular Biology Laboratory and SwissProt databases. The fact that MM-2 shares several amino acids with other peptides that accumulated in the selection suggests a common motif. However, MM-2 might not contain the optimal binding motif since the other peptides share amino acids with each other but not with MM-2, pointing toward possible improvements of the binding motif. Therefore, modifications of the lead sequence in MM-2 should result in peptides with improved targeting properties. In biodistribution studies, the peptide showed a loss of accumulation in the tumour as well as in the other organs with time, although the decrease in tumour was slower than in organs. This leads to an increase of tumour to organ ratios for all organs. The decrease of binding observed could indicate degradation of the peptide in vivo despite the observed serum stability. Alternatively, deiodination may occur while the peptide is still intact.

Bacterial peptide display can be used to identify stable peptides for tumour targeting. This technology is less time consuming since phage precipitation and titering can be avoided and the handling of bacteria is easier as compared to the phage display technology. The thioredoxin part leads to a stable conformation, and cyclization enhances the serum stability of the resulting peptides. Furthermore, the thioredoxin loop confers a greater versatility for peptide integration into surface structures without changing peptide conformation as it would be the case for N-terminal expressed phage display peptides. However, as shown in the case of MM-2, stability in serum does not have to result in stability in vivo, and further modifications such as changes in the peptide sequence are needed. Imaging has not been done with molecules identified using this technique.

\section{Ribosome and mRNA display}

In vitro selection systems such as ribosome display and mRNA display, although technically demanding, have two important advantages: the ability to handle very large libraries $\left(10^{12}-10^{13}\right.$ different sequences) and the possibility of using polymerase chain reaction (PCR) amplification steps to introduce further diversity into the system, which may be used to evolve proteins through an iteration of random mutagenesis and selection (affinity maturation) [46, 47]. Both ribosome display and mRNA display have been used to select linear peptides or single-chain antibodies that bind to protein targets with low picomolar affinities.

Ribosome display is based on the translation of a library consisting of mRNA molecules with a stoichiometric quantity of ribosomes. The mRNAs in the library used has no stop codon, which extends the scanning process of the ribosome to the end of the mRNA molecule. The corresponding polypeptide emerges from the ribosome while its end is still fixed within the ribosomal tunnel, and its last amino acid is connected to the peptidyl-tRNA. The absence of stop codons prevents the binding of release factors, which normally catalyse the release of the polypeptide from the ribosome. This leads to the formation of a protein-ribosome-RNA complex, which connects the phenotype to the genotype [47, 48]. The different steps of ribosome display are: construction of a large DNA library encoding the polypeptide of interest fused in frame to a Cterminal spacer, in vitro transcription of the library into mRNA and in vitro translation. Thereafter, the complexes consisting of protein, mRNA and ribosomes are exposed to the target structure including washing steps to remove nonbinders leading to an enrichment of binding molecules. Amplification is done by reverse transcriptase PCR (RTPCR), followed by the next round of transcription, translation and exposure. During this amplification step diversity may be further increased using error-prone PCR. Identification of high-affinity binders is usually obtained after three to six rounds.

Random libraries were used to select peptides for binding to several targets such as an antibody against dynorphin B, the prostate-specific antigen, streptavidin and 
lysozyme or to identify the main antigenic polypeptides of Staphylococcus aureus. The peptides ranged in affinity from 7.2 to $140 \mathrm{nM}$. Similar results were obtained for $\mathrm{scFv}$ fragments using targets such as the GCN4 leucine zipper, DNA structures in eukaryotic telomeres, progesterone and fluorescein. In this setting all selected scFvs acquired genetic mutations during the cycles of ribosome display. A biophysical comparison of the isolated scFvs with their progenitors revealed that all selected scFvs showed mutations due to errors introduced by the DNA polymerase and that these mutations led to significant improvement (by up to 40 -fold) in their affinities to the antigen. The best scFvs selected had affinities in the low picomolar range and could be further improved by off-rate selection and error-prone PCR [49].

mRNA display uses a similar strategy to ribosome display: a complex between mRNA and the polypeptide encoded by the mRNA can be applied in a specific selection process [50, 51]. The procedure differs from ribosome display in the covalent nature of the linkage between the mRNA and the protein in the mRNA-protein complex. This is achieved by linking the two molecules through a small adaptor molecule, typically puromycin. The different steps include transcription of a large DNA library encoding the molecules of interest and free of stop codons into mRNA, ligation of an adaptor molecule to the $3^{\prime}$ ends and in vitro translation. This procedure results in a peptide bond between the adaptor molecule and the C-terminal amino acid residue in the polypeptide chain. After introduction of cDNA chains by reverse transcription for stabilization and ease of recovery of the genetic information, the cDNA/mRNA protein library is exposed to the target. Isolation of binders is done by affinity chromatography or immunoprecipitation of the target structure. The cDNA is then used for further amplification and the next rounds of panning. Four to ten rounds of selection may be necessary to select proteins with nanomolar affinity for a given target, as has been done with engineered libraries of linear peptides, constrained peptides, single-domain antibody mimics, variable heavy domains of antibodies and single-chain antibodies. Using targets such as an anti-c-Myc antibody or streptavidin binders with a $\mathrm{K}_{\mathrm{D}}$ down to $2.5 \mathrm{nM}$ were found [52-54].

\section{Molecular evolution-DNA shuffling}

Darwinian design of molecules can be attributed to a mindless algorithm of mutation and selection iterated over a long period of time [55]. This strategy of nature has been copied by molecular biology techniques with the in vitro evolutionary process being much faster. Protein engineering usually proceeded by modelling of individual changes followed by site-directed mutagenesis of the corresponding DNA with a designed oligonucleotide primer, an expression of the recombinant protein in mutants for testing and evaluation. However, this technique is not able to create many novel biomolecules simultaneously. To enhance the biomolecular diversity, new strategies have been used such as repeated cycles of "error-prone PCR" [56] and repeated oligonucleotide-directed mutagenesis [57]. Error-prone PCR employs a low-fidelity replication step to introduce random point mutations at each round of amplification. This method has the advantage of being simple and easy to use, but its power is also limited as a result of the small sizes of libraries relative to the sequence size.

Besides site-directed mutagenesis and error-prone PCR directed evolution has been applied to mimic Darwinian evolution. The method of directed (molecular) evolution was initially developed for creating RNA with novel binding and catalytic activities. DNA shuffling mimics natural recombination by allowing in vitro homologous recombination of DNA [58]. Therefore, a population of related genes is randomly fragmented by DNAse I and subjected to denaturation and hybridization, followed by the extension of $5^{\prime}$ overhang fragments by Taq DNA polymerase. A DNA recombination occurs when a fragment derived from one template primes a template with a combination of overlapping and different sequences. The reassembled gene library is subjected to selection for individual variants with specific properties. These are used for further cycles of mutation, shuffling and amplification. For each cycle the selection pressure applied to screen the library leads to an accumulation of beneficial mutations with evolution of the best-fit molecule. This concentration of beneficial mutations under selection pressure is uniquely found only in the biological approach, and not by the use of chemical methods. However, this also implies that the development of assays for functional screening is a critical step to ensure that the mutations accumulated in each successive cycle are directed specifically toward the defined property. Possible applications of this method include the improvement of enzyme properties, development of altered metabolism pathways, antibiotics and pharmaceutically active proteins, development of plasmids or viruses for novel vaccines and gene therapy applications. Genes from multiple parents and even from different species can be shuffled in a single step in operations that do not occur in nature but may be very useful for the development of diagnostic and therapeutic approaches. Although this technology has not been applied yet for the development of imaging tracers we conceive that it bears potential for the generation of high-affinity binders.

\section{Conclusions}

The techniques mentioned are all artificial in that they create (1) a variety of species and (2) a specific selection 
pressure. The first may occur in the setting of a specifically designed structure, the second is driven by the researchers' intention to finally produce molecules with desired properties. Using these novel biotechnological procedures, a lot of nonsense is created due to their high throughput nature. This is especially the case for DNA shuffling. Therefore, the selection process is critical in these cases, heavily depending on the read-out system applied to isolate the desired species (molecules). For most DNA shuffling experiments this is done with enzyme activity assays, but also a combination with display techniques may be used for the generation of novel receptor ligands.

Although it may be thought that there is a considerable "creationist" component in these techniques, it has to be mentioned that this only applies to the design of the starting point of these experiments. The mechanisms at work during the generation of a variety of species and the process of selection are purely evolutionary.

Coming back to the initial question about the Darwinian nature of molecular imaging today, we realize that in daily practice we still rely on conventional tracers which are designed by modification of known substrates, ligands or other molecules. However, high throughput methods for ligand selection enter the field which add new classes of molecules with improved properties with respect to stability, clearance, transport and affinity. A significant amount of time and efforts from molecular imaging scientists will be necessary before we can consider Darwinian molecular imaging as useful for daily practice. In between undue optimism and excessive pessimism, we may remember that, from his first insight during his voyage on the Beagle as a young man and its publication, it took Charles Darwin 25 years to carefully mature his theory of evolution and challenge the theological myth of the perfection of creation.

\section{References}

1. Darwin C. The origin of species. London: Penguin; 1968.

2. Dobzhansky T. Nothing in biology makes sense except in the light of evolution. The American Biology Teacher 1973;35:125-9.

3. Darwin C. On the origin of species by means of natural selection, or the preservation of favoured races in the struggle for life. London: John Murray; 1859.

4. De Hevesy G. Some applications of isotopic indicators. Nobel Lecture, 12 December 1944. Available via http://nobelprize.org/ nobel_prizes/chemistry/laureates/1943/hevesy-lecture.pdf.

5. Schrödinger E. What is life? The physical aspect of the living. New York: The Macmillan Company; 1946.

6. Joyce GF. Directed molecular evolution. Sci Am 1992;267(6):907.

7. Gold L, Polisky B, Uhlenbeck O, Yarus M. Diversity of oligonucleotide functions. Annu Rev Biochem 1995;64:763-97.

8. Robertson DL, Joyce GF. Selection in vitro of an RNA enzyme that specifically cleaves single-stranded DNA. Nature 1990;344: 467-8.
9. Watson JD, Crick FH. Molecular structure of nucleic acids: a structure for deoxyribose nucleic acid. Nature 1953;171:7378.

10. Green R, Ellington AD, Szostak JW. In vitro genetic analysis of the Tetrahymena self-splicing intron. Nature 1990;347:406-8.

11. Blackwell TK, Kretzner L, Blackwood EM, Eisenman RN, Weintraub H. Sequence-specific DNA binding by the c-Myc protein. Science 1990;250:1149-51.

12. Blackwell TK, Weintraub H. Differences and similarities in DNAbinding preferences of $\mathrm{MyoD}$ and $\mathrm{E} 2 \mathrm{~A}$ protein complexes revealed by binding site selection. Science 1990;250:1104-10.

13. Ellington AD, Szostak JW. In vitro selection of RNA molecules that bind specific ligands. Nature 1990;346:818-22.

14. Tuerk C, Gold L. Systematic evolution of ligands by exponential enrichment: RNA ligands to bacteriophage T4 DNA polymerase. Science 1990;249:505-10.

15. Carothers JM, Szostak JW. In vitro selection of functional oligonucleotides and the origins of biochemical activity. In: Klussmann S, editor. The aptamer handbook. Weinheim, Germany: Wiley-VCH Verlag GmbH \& Co. KgaA; 2006. p. 3-28.

16. Hamaguchi N, Ellington A, Stanton M. Aptamer beacons for the direct detection of proteins. Anal Biochem 2001;294:126-31.

17. Babendure JR, Adams SR, Tsien RY. Aptamers switch on fluorescence of triphenylmethane dyes. J Am Chem Soc 2003; 125:14716-7.

18. Babendure JR, Babendure JL, Ding JH, Tsien RY. Control of mammalian translation by mRNA structure near caps. RNA 2006;12:851-61.

19. Werstuck G, Green MR. Controlling gene expression in living cells through small molecule-RNA interactions. Science 1998;282:296-8.

20. Bagalkot V, Zhang L, Levy-Nissenbaum E, Jon S, Kantoff PW, Langer R, et al. Quantum dot-aptamer conjugates for synchronous cancer imaging, therapy, and sensing of drug delivery based on bifluorescence resonance energy transfer. Nano Lett 2007;7:306570.

21. Charlton J, Sennello J, Smith D. In vivo imaging of inflammation using an aptamer inhibitor of human neutrophil elastase. Chem Biol 1997;4:809-16.

22. Hicke BJ, Stephens AW, Gould T, Chang YF, Lynott CK, Heil J, et al. Tumor targeting by an aptamer. J Nucl Med 2006;47:668-78.

23. Gambhir SS. Using radiolabeled DNA as an imaging agent to recognize protein targets. J Nucl Med 2006;47:557-8.

24. McCauley TG, Kurz JC, Merlino PG, Lewis SD, Gilbert M, Epstein DM, et al. Pharmacologic and pharmacokinetic assessment of anti-TGFbeta2 aptamers in rabbit plasma and aqueous humor. Pharm Res 2006;23:303-11.

25. Smith GP. Filamentous fusion phage: novel expression vectors that display cloned antigens on the virion surface. Science 1985;228:1315-7.

26. Smith GP, Petrenko VA. Phage display. Chem Rev 1997;97:391410 .

27. Koivunen E, Arap W, Valtanen H, Rainisalo A, Medina OP, Heikkilä $\mathrm{P}$, et al. Tumor targeting with a selective gelatinase inhibitor. Nat Biotechnol 1999;17:768-74.

28. Pasqualini R. Vascular targeting with phage peptide libraries. Q J Nucl Med 1999;43:159-62.

29. Burg MA, Pasqualini R, Arap W, Ruoslahti E, Stallcup WB. NG2 proteoglycan-binding peptides target tumor neovasculature. Cancer Res 1999;59:2869-74.

30. Oyama T, Sykes KF, Samli KN, Minna JD, Johnston SA, Brown KC. Isolation of lung tumor specific peptides from a random peptide library: generation of diagnostic and cell-targeting reagents. Cancer Lett 2003;202:219-30.

31. Zitzmann S, Mier W, Schad A, Kinscherf R, Askoxylakis V, Krämer S, et al. A new prostate carcinoma binding peptide (DUP- 
1) for tumor imaging and therapy. Clin Cancer Res 2005;11:13946.

32. Askoxylakis V, Zitzmann S, Mier W, Graham K, Krämer S, von Wegner F, et al. Preclinical evaluation of the breast cancer cellbinding peptide, p160. Clin Cancer Res 2005;11:6705-12.

33. Askoxylakis V, Mier W, Zitzmann S, Ehemann V, Zhang J, Kramer S, et al. Characterization and development of a peptide (p160) with affinity for neuroblastoma cells. J Nucl Med 2006;47:981-8.

34. Zitzmann S, Krämer S, Mier W, Hebling U, Altmann A, Rother A, et al. Identification and evaluation of a new tumor cell-binding peptide, FROP-1. J Nucl Med 2007;48:965-72.

35. Ryu DD, Nam DH. Recent progress in biomolecular engineering. Biotechnol Prog 2000;16:2-16.

36. Viti F, Tarli L, Giovannoni L, Zardi L, Neri D. Increased binding affinity and valence of recombinant antibody fragments lead to improved targeting of tumoral angiogenesis. Cancer Res 1999;59:347-52.

37. Santimaria M, Moscatelli G, Viale GL, Giovannoni L, Neri G, Viti $\mathrm{F}$, et al. Immunoscintigraphic detection of the ED-B domain of fibronectin, a marker of angiogenesis, in patients with cancer. Clin Cancer Res 2003;9:571-9.

38. Rossin R, Berndorff D, Friebe M, Dinkelborg LM, Welch MJ. Small-animal PET of tumor angiogenesis using a (76)Br-labeled human recombinant antibody fragment to the ED-B domain of fibronectin. J Nucl Med 2007;48:1172-9.

39. Berndorff D, Borkowski S, Moosmayer D, Viti F, Müller-Tiemann $\mathrm{B}$, Sieger S, et al. Imaging of tumor angiogenesis using $99 \mathrm{mTc}-$ labeled human recombinant anti-ED-B fibronectin antibody fragments. J Nucl Med 2006;47:1707-16.

40. El-Emir E, Dearling JL, Huhalov A, Robson MP, Boxer G, Neri D, et al. Characterisation and radioimmunotherapy of L19-SIP, an anti-angiogenic antibody against the extra domain $\mathrm{B}$ of fibronectin, in colorectal tumour models. Br J Cancer 2007;96:1862-70.

41. Deutscher SL, Figueroa SD, Kumar SR. Tumor targeting and SPECT imaging properties of an (111)In-labeled galectin-3 binding peptide in prostate carcinoma. Nucl Med Biol 2009;36:137-46.

42. Nothelfer EM, Zitzmann-Kolbe S, Garcia-Boy R, Krämer S, Herold-Mende C, Altmann A, et al. Identification and characterization of a peptide with affinity to head and neck cancer. J Nucl Med 2009;50:426-34.

43. Lu Z, Murray KS, Van Cleave V, LaVallie ER, Stahl ML, McCoy JM. Expression of thioredoxin random peptide libraries on the Escherichia coli cell surface as functional fusions to flagellin: a system designed for exploring protein-protein interactions. Biotechnology (N Y) 1995; 13:366-72.

44. Thai CK, Dai H, Sastry MS, Sarikaya M, Schwartz DT, Baneyx F. Identification and characterization of $\mathrm{Cu}(2) \mathrm{O}-$ and $\mathrm{ZnO}$-binding polypeptides by Escherichia coli cell surface display: toward an understanding of metal oxide binding. Biotechnol Bioeng 2004;87:129-37.

45. Zitzmann S, Krämer S, Mier W, Mahmut M, Fleig J, Altmann A, et al. Identification of a new prostate-specific cyclic peptide with the bacterial FliTrx system. J Nucl Med 2005;46:782-5.

46. Mattheakis LC, Bhatt RR, Dower WJ. An in vitro polysome display system for identifying ligands from very large peptide libraries. Proc Natl Acad Sci U S A 1994;91:9022-6.

47. Lipovsek D, Plückthun A. In-vitro protein evolution by ribosome display and mRNA display. J Immunol Methods 2004;290:51-67.

48. Zahnd C, Amstutz P, Plückthun A. Ribosome display: selecting and evolving proteins in vitro that specifically bind to a target. Nat Methods 2007;4:269-79.

49. Hanes J, Schaffitzel C, Knappik A, Plückthun A. Picomolar affinity antibodies from a fully synthetic naive library selected and evolved by ribosome display. Nat Biotechnol 2000;18: 1287-92.

50. Roberts RW, Szostak JW. RNA-peptide fusions for the in vitro selection of peptides and proteins. Proc Natl Acad Sci U S A 1997;94:12297-302.

51. Keefe AD, Szostak JW. Functional proteins from a randomsequence library. Nature 2001;410:715-8.

52. Baggio R, Burgstaller P, Hale SP, Putney AR, Lane M, Lipovsek $\mathrm{D}$, et al. Identification of epitope-like consensus motifs using mRNA display. J Mol Recognit 2002;15:126-34.

53. Chan S, Gabra H, Hill F, Evan G, Sikora K. A novel tumour marker related to the c-myc oncogene product. Mol Cell Probes 1987;1:73-82.

54. Wilson DS, Keefe AD, Szostak JW. The use of mRNA display to select high-affinity protein-binding peptides. Proc Natl Acad Sci U S A 2001;98:3750-5.

55. Dennett DC. Darwin's dangerous idea. New York: Simon and Schuster; 1995.

56. Cadwell RC, Joyce GF. Mutagenic PCR. PCR Methods Appl 1994;3:S136-40.

57. Reidhaar-Olson J, Bowie J, Breyer RM, Hu JC, Knight KL, Lim WA, et al. Random mutagenesis of protein sequences using oligonucleotide cassettes. Methods Enzymol 1991;208:564-86.

58. Stemmer WP. Rapid evolution of a protein in vitro by DNA shuffling. Nature 1994;370:389-91. 